\title{
Tratamiento Superficial de Acero Galvanizado con Películas Híbridas formadas por 3-(trimetoxisililpropil) metacrilato (TMSPMA) y Tetraetoxisilano (TEOS)
}

\author{
Sandra R. Kunst, José F. Matos, Elen A. Leal da Silva y Célia F. Malfatti \\ Universidade Federal do Rio Grande do Sul, Escuela de Ingeniería, Departamento de Metalurgia, \\ Porto Alegre-Brasil (e-mail:celia.malfatti@ufrgs.br)
}

Recibido Abr. 18, 2011; Aceptado May. 26, 2011; Versión Final recibida Ago. 04, 2011

\begin{abstract}
Resumen
Acero galvanizado fue revestido con una película híbrida a partir de una solución formada por los precursores silanos 3-(trimetoxisililpropil) metacrilato (TMSPMA) y tetraetoxisilano (TEOS) con adición de cerio. El uso de capas hibridas orgánico-inorgánico representa una alternativa ambientalmente aceptable para mejorar la resistencia de una serie de materiales metálicos frente a los procesos corrosivos. Se emplearon tres tiempos de inmersión (2, 10 y 15 minutos) y se caracterizaron las películas mediante microscopia electrónica de barrido, evaluando también el carácter hidrofóbico de las películas. El comportamiento electroquímico de los revestimientos obtenidos fue determinado por el monitoreo del potencial de circuito abierto, polarización potenciodinámica e impedancia electroquímica. Los resultados que se obtuvo evidenciaron el efecto del tiempo de permanencia dentro de la solución en la uniformidad de la película y consecuentemente sobre la resistencia a la corrosión del mismo.
\end{abstract}

\section{Surface Treatment of Galvanized Steel with Hybrid Films formed by 3-(trimethoxysilyl) methacrylate (TMSPMA) and Tetraethoxysilane (TEOS)}

\begin{abstract}
Galvanized steel was coated with a hybrid film obtained from a solution formed by silane precursor 3-( trimethoxysilyl) methacrylate (TMSPMA) and tetraethoxysilane (TEOS) with addition of cerium. The use of hybrid organic-inorganic layers represents an environmentally friendly alternative to improve resistance against corrosion of a series of metallic materials. Three immersion times (2, 10 and 15 minutes) were used and the films were characterized by scanning electron microscope evaluating film wetability was at the same time. The electrochemical behavior of the coatings obtained was evaluated by open circuit potential, potentiodynamic polarization and electrochemical impedance. The results showed the effect of immersion time in the solution on the film uniformity and consequently on the corrosion resistance of these films.
\end{abstract}

Keywords: silane, immersion time, corrosion, TMSPMA, TEOS 


\section{INTRODUCCION}

Los pre-tratamientos como el cromado y el fosfatado han sido utilizados por la industria como métodos eficientes en la prevención de la corrosión para el acero galvanizado y también por sus excelentes características de adhesión. Por lo tanto, estos procesos aún siendo muy funcionales son cancerígenos y producen residuos tóxicos al medio ambiente. Por este motivo estos tratamientos son sometidos a drásticas regulaciones gubernamentales de los órganos pertinentes de protección al medio ambiente (Cabral, 1979). Debido a esto, se han desarrollado durante los últimos años tratamientos alternativos que no sean tóxicos ni cancerígenos y que puedan sustituir a los procesos antes mencionados de una forma eficiente. Entre estas alternativas los pretratamiento basados en silanos (funcionales y organofuncionales) han demostrado resultados excelentes, atrayendo de esa manera la atención del sector industrial en los últimos años, debido a que los silanos mejoran las características de protección contra la corrosión del substrato y las propiedades de adhesión de los revestimientos orgánicos, además de provocar impactos ambientales muy inferiores, comparados a los procesos de cromado y fosfatado (Jones, 1996; Epa, 1998). Las películas de silanos y las películas híbridas actúan como un revestimiento barrera retardando la penetración de las especies agresivas hasta el substrato metálico, promoviendo de esa forma la protección contra la corrosión. Los grupos inorgánicos presentan características de materiales cerámicos contribuyendo al aumento de la resistencia al desgaste (Trabelsi, 2004).

El resultado del revestimiento puede ser mejorado por la adición de inhibidores de corrosión. En los estudios recientes se demostró que los cationes de las tierras raras pueden otorgar propiedades anticorrosivas comparables a los cromatos (Arenas, 2003). Dentro de los posibles aditivos se encuentran las sales de cerio y lantano que presentan excelentes propiedades para inhibir el proceso de corrosión del acero (Bernal, 1995), acero galvanizado (Aramaki, 2001), del aluminio y sus aleaciones (Aldykiewicza, 1996). La resistencia a la corrosión producida por el cerio puede ser atribuida a la formación y precipitación de óxidos o hidróxidos de cerio en los sitios catódicos (Montemor, 2002), de forma alternada promoviendo una reducción de la taza de reacciones catódicas aumentando la resistencia a la corrosión del material (Geary, 1997).

Existen varias técnicas para obtener revestimientos de silano (dip coating, spin coating y spray coating), que se fundamentan en la hidrólisis y condensación de precursores moleculares (Montemor, 2006). El proceso más usado es el dip coating, que consiste en la inmersión y retirada del substrato en la solución que contiene precursores de silano a velocidad controlada, el espesor de la película va a ser función de las siguientes variables, velocidad de inmersión y retirada, acabado superficial y viscosidad de la solución (Ooij, 2005; Yasakau, 2007).

El objetivo de este trabajo fue proponer una pre-tratamiento ecológicamente correcto, para sustituir al cromado y evitar la producción de $\mathrm{Cr}^{+6}$, que es tóxico y cancerígeno. En los experimentos realizados se pude ver, que el tiempo de inmersión en la solución de silano influye en la uniformidad de la película formada. El uso de soluciones de silano con bajos tiempos de inmersión, además de formar una película heterogénea, también afecta los resultados cuando los experimentos se repiten y se realiza la caracterización de la capa (Kunst, 2011). El acero galvanizado fue revestido con una película de silano obtenida a partir de un sol constituido por los precursores silanos 3-(trimetoxisililpropil) metacrilato (TMSPMA) y Tetraetoxisilano (TEOS) con una adición de nitrato de cerio a una concentración de 0,01 mol/L, empleando diferentes tiempos de inmersión (2, 10 y 15 minutos) y una velocidad de retirada de $20 \mathrm{~cm} / \mathrm{min}$. La caracterización de las películas fue realizada por MEB, además las películas fueron evaluadas con respecto a su hidrofobicidad. El comportamiento electroquímico de los revestimientos obtenidos fue calculado por el potencial del circuito abierto, polarización potenciodinámica e impedancia electroquímica.

\section{MATERIALES Y MÉTODOS}

\section{Preparación de la superficie}

Los substratos de acero galvanizado fueron lavados con etanol y secados con aire caliente, luego fueron desengrasados con detergente neutro $(\mathrm{pH}=7)$ a una temperatura de $70^{\circ} \mathrm{C}$ y con un tiempo 
de inmersión de 10 minutos. En seguida fueron lavados con agua desionizada y secadas nuevamente para luego ser lavado con etanol y finalmente secados con aire caliente.

Elaboración de las películas de silano

Las reacciones de hidrólisis de los silanos (TMSPMA) 3-(trimetoxisililpropil) metacrilato $\left(\mathrm{C}_{10} \mathrm{H}_{20} \mathrm{SiO}_{5}\right)$ y (TEOS) Tetraetoxisilano $\left(\mathrm{C}_{8} \mathrm{H}_{20} \mathrm{SiO}_{4}\right)$, alcohol y agua, fueron colocadas en el siguiente orden silano/alcohol/agua, obteniendo una solución a la cual se adicionó nitrato de cerio con una concentración de $0.01 \mathrm{M}$. Luego de 24 horas de hidrólisis de la solución se tuvo un valor de $\mathrm{pH}$ final de 4,75 .

La aplicación de las soluciones conteniendo los silanos hidrolizados fue realizada por el proceso de revestimiento por inmersión con velocidad de retirada de $20 \mathrm{~cm} / \mathrm{min}$ y a diferentes tiempos de permanencia en la solución (2,10, y 15 minutos). Luego del proceso de inmersión en caliente los substratos pre-tratados con películas de silano fueron curados empleando el proceso de curado térmico a la temperatura de $60^{\circ} \mathrm{C} \pm 2$ por 20 minutos en una estufa. La tabla 1 , presenta la descripción de las muestras utilizadas.

Tabla 1: Descripción de las muestras utilizadas.

\begin{tabular}{|l|l|}
\hline Muestra & Descripción \\
\hline AG & Acero galvanizado sin película de silano \\
\hline AG/Sol-gel2 & $\begin{array}{l}\text { Acero galvanizado revestido con película de silano y con un tiempo de permanencia } \\
\text { en la solución de } 2 \text { minutos }\end{array}$ \\
\hline AG/Sol-gel10 & $\begin{array}{l}\text { Acero galvanizado revestido con película de silano y con un tiempo de permanencia } \\
\text { en la solución de } 10 \text { minutos }\end{array}$ \\
\hline AG/Sol-gel15 & $\begin{array}{l}\text { Acero galvanizado revestido con película de silano y con un tiempo de permanencia } \\
\text { en la solución de 15 minutos }\end{array}$ \\
\hline
\end{tabular}

\section{Caracterización de los revestimientos}

Caracterización morfológica: El análisis morfológico de la superficie fue realizado por MEB. EI equipo usado fue JEOL-JSM 5800 Scanning Microscope, con tensión de aceleración de $20 \mathrm{keV}$.

Caracterización electroquímica: Para la investigación electroquímica fue utilizado un potenciostato (Omnimetra Mod.PG-05) acoplado a un analizador de respuesta en frecuencia (Solartron 1255), para obtener las medidas de impedancia electroquímica. Los ensayos fueron realizados en una celda electroquímica de tres electrodos donde se utilizó como electrodo de trabajo las muestras de AG, AG/Sol-gel2, AG/Sol-gel10, AG-Sol-gel15 (área aprox. $0.626 \mathrm{~cm}^{2}$ ), el electrodo de referencia fue calomelano saturado (ECS) y como contra-electrodo un alambre de platino.

El electrolito usado fue el $\mathrm{NaCl} 5 \%$, el potencial de circuito abierto fue monitoreado durante la primera hora de inmersión de las muestras en el electrolito. Las medidas de espectroscopia de impedancia electroquímica (EIS), fueron hechas en el potencial de circuito abierto en la franja de frecuencia de $100 \mathrm{kHz}$ a $0,01 \mathrm{~Hz}$, con una amplitud de señal senoidal de $10 \mathrm{mV}$. Las curvas de polarización fueron calculadas con velocidades de barrido de $1 \mathrm{mV} / \mathrm{s}$ en una franja de potencial de $200 \mathrm{mV}$ (abajo del potencial de circuito abierto) hasta $1000 \mathrm{mV}$ (encima del potencial de circuito abierto).

\section{Angulo de contacto}

La prueba fue realizada por el método de la gota sésil a partir de un equipo desarrollado por el Laboratorio de Optica y Laser del Instituto de Física de la UFRGS. El ángulo de contacto se determinó por medio de un programa de análisis de imágenes, se realizaron cuatro mediciones para cada muestra. 


\section{RESULTADOS}

\section{Caracterización morfológica}

La figura 1, muestra las micrografías obtenidas por MEB, para todos los sistemas estudiados AG, AG/Sol-gel2, AG/Sol-gel10, AG-Sol-gel15, luego del proceso de revestimiento por inmersión y curado de los sistemas.
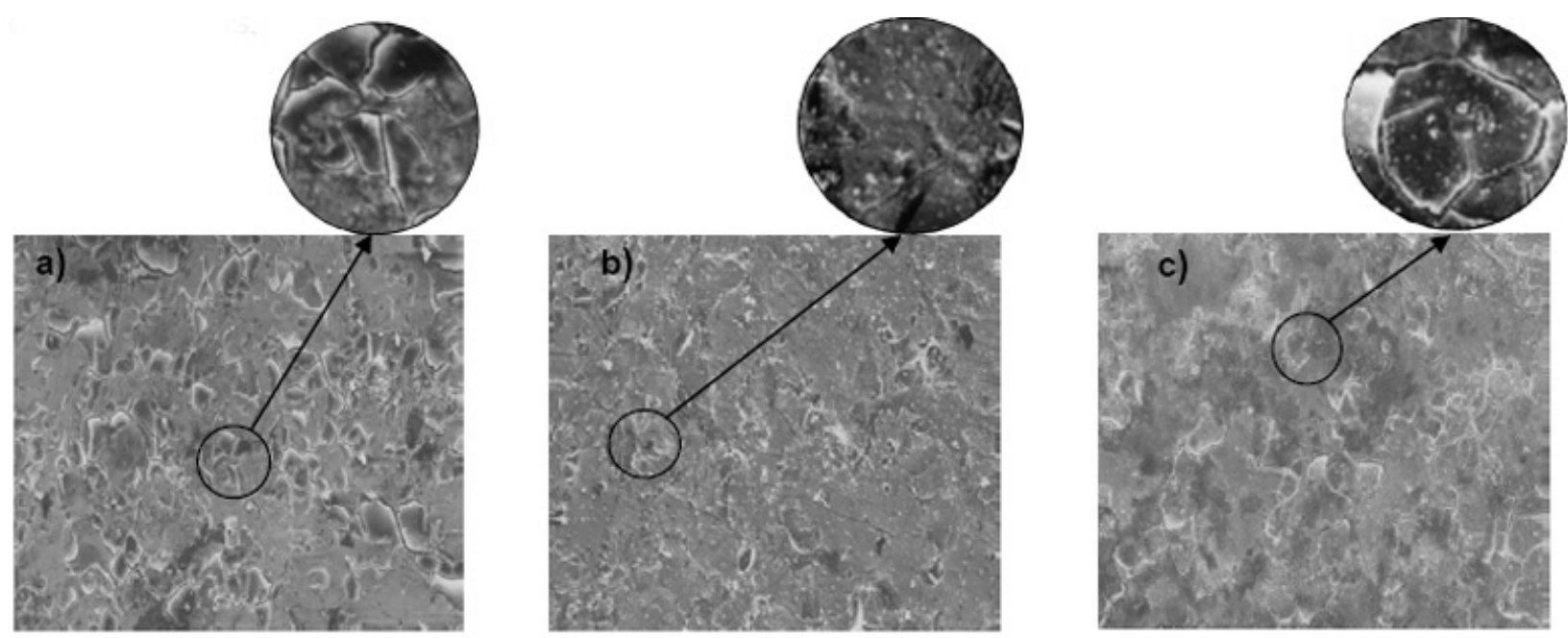

Fig. 1: Micrografias obtenidas por MEB, para todos los sistemas estudiados (a) AG/Sol-gel2, (b) AG/Sol-gel10, (c) AG/Sol-gel15

\section{Angulo de contacto}

La figura 2, muestra las imágenes obtenidas para la determinación del ángulo de contacto de contacto por el método de la gota sésil. A partir de la tabla 2, es posible observar las medidas del ángulo de contacto obtenidos para los sistemas estudiados.
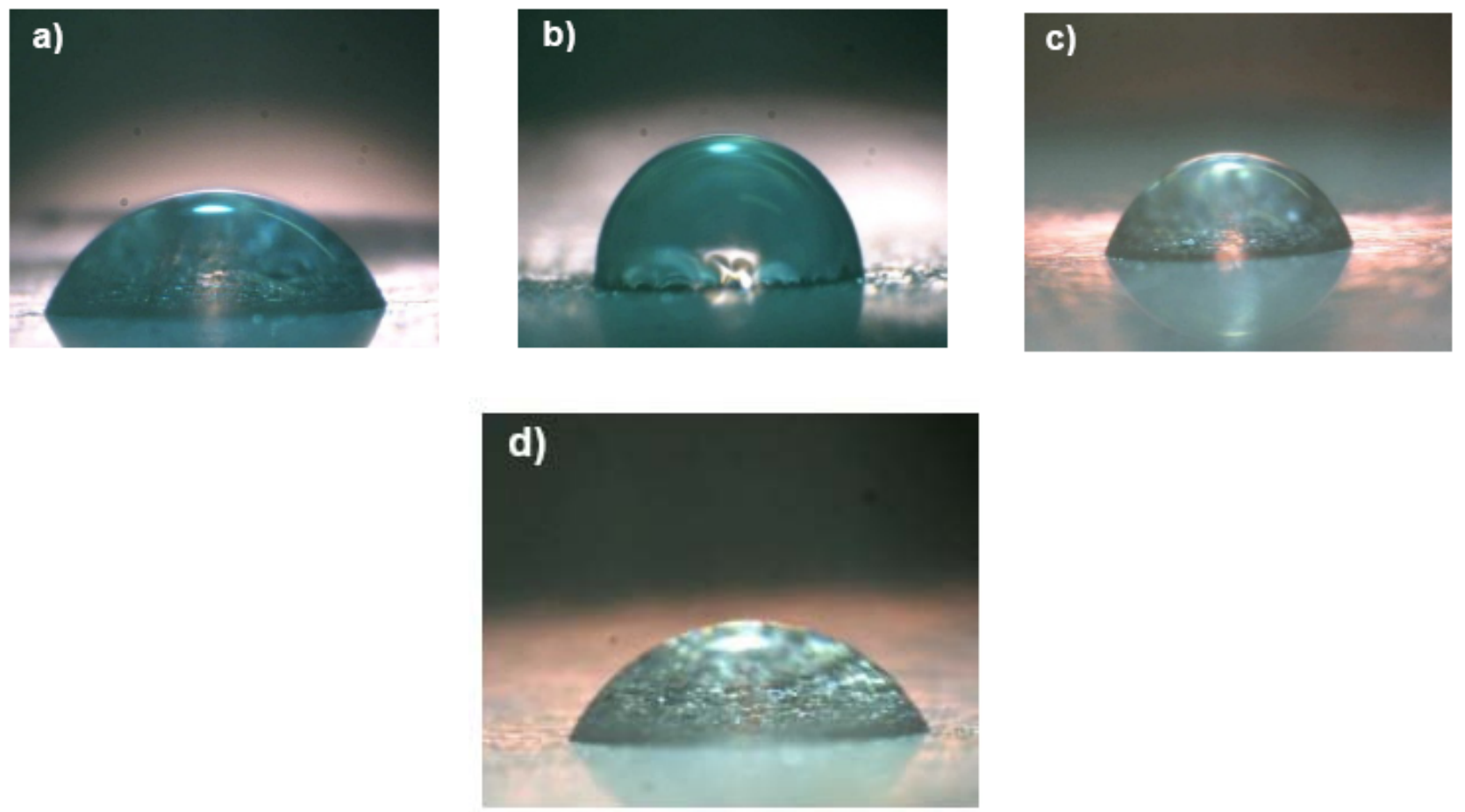

Fig. 2: Imágenes obtenidas para la determinación para el ángulo de contacto para los sistemas (a) AG/Sol-gel2, (b) AG/Sol-gel10, (c) AG/Sol-gel15, (d) AG 
Tabla 2: Angulo de contacto medido con la desviación del padrón obtenido por el método de la gota sésil

\begin{tabular}{|c|c|}
\hline Muestra & Angulo de contacto \\
\hline AG/Sol-gel2 & $67^{\circ} \pm 1,6$ \\
\hline AG/Sol-gel10 & $92^{\circ} \pm 0,9$ \\
\hline AG/Sol-gel15 & $76^{\circ} \pm 0,4$ \\
\hline AG & $66^{\circ} \pm 1,1$ \\
\hline
\end{tabular}

\section{Caracterización Electroquímica}

Potencial de circuito abierto

Fueron realizados medidas de potencial de circuito abierto a fin de monitorear la variación del potencial con respecto al tiempo de inmersión (figura 3).

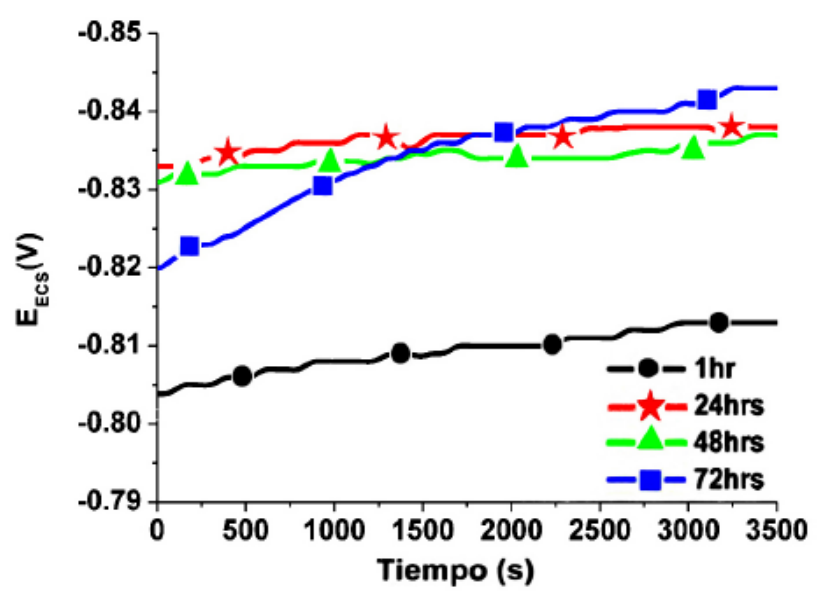

Fig. 3: Curvas de potencial de circuito abierto obtenidas para los sistemas estudiados

\section{Curvas de polarización}

A partir de la extrapolación de las rectas de Tafel, aplicada a las curvas de la polarización presentadas la figura 4, fueron determinados el potencial de corrosión (Ecorr), la densidad de corriente de corrosión (icorr) y la resistencia de polarización (Rp) para todos los sistemas estudiados (tabla 3 ).

Tabla 3: Datos obtenidos a partir de la extrapolación de las rectas de Tafel

\begin{tabular}{|c|c|c|c|}
\hline Muestra & $\mathrm{i}_{\text {corr }}\left(\mathrm{A} / \mathrm{cm}^{2}\right)$ & $\mathrm{E}_{\text {corr }}(\mathrm{mV})$ & $\operatorname{Rp}\left(\Omega / \mathrm{cm}^{2}\right)$ \\
\hline AG/Sol-gel2 & $9,09 \times 10^{-6}$ & -846 & $2,86 \times 10^{3}$ \\
\hline AG/Sol-gel10 & $2,36 \times 10^{-6}$ & -847 & $9,18 \times 10^{3}$ \\
\hline AG/Sol-gel15 & $6,38 \times 10^{-6}$ & -850 & $4,08 \times 10^{3}$ \\
\hline AG & $2,65 \times 10^{-6}$ & -820 & $9,80 \times 10^{2}$ \\
\hline
\end{tabular}




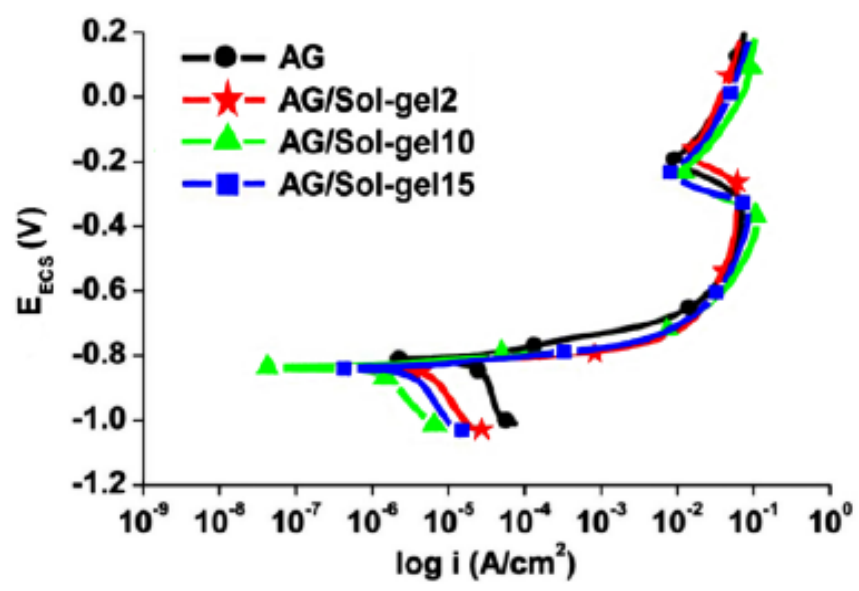

Fig. 4: Curvas de polarización obtenidas para los sistemas estudiados.

Las imágenes obtenidos por MEB, para todos los sistemas estudiados AG/Sol-gel2, AG/Sol-gel10 y AG/Sol-gel15, luego del ensayo de polarización se muestra en la figura 5.
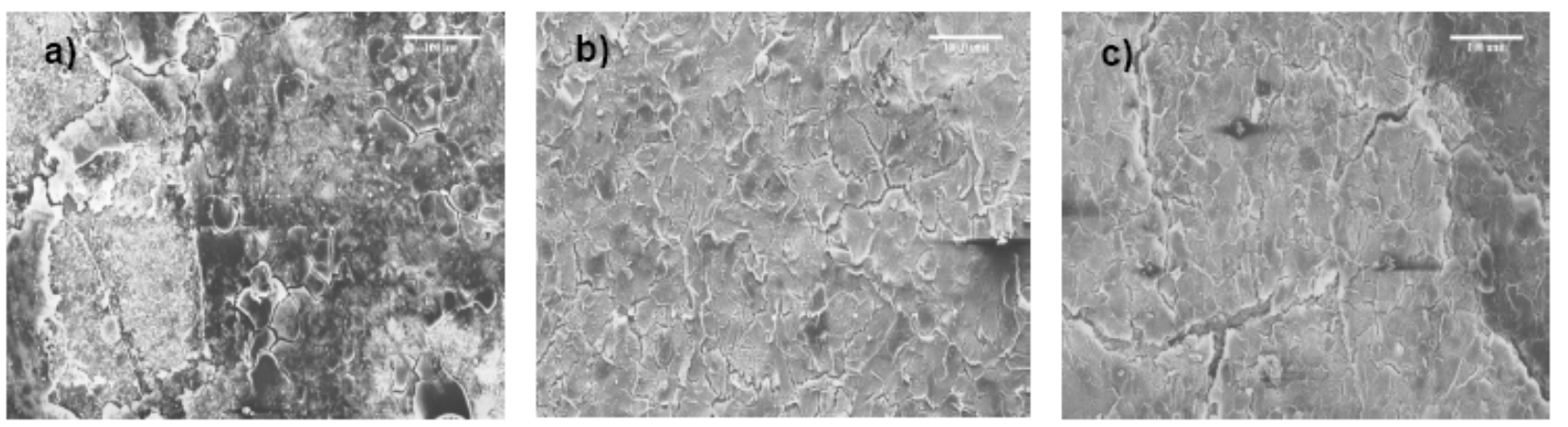

Fig. 5: Micrografías obtenidas por MEB, luego del ensayo de polarización para los sistemas, (a) AG/Sol-gel2, (b) AG/Sol-gel10 y (c) AG/Sol-gel15

\section{Espectroscopia de impedancia electroquímica}

Los diagramas de Bode fueron obtenidos por ensayo de impedancia electroquímica realizados durante 72 horas de inmersión y los resultados se presentan en la figura 6.

\section{DISCUSION}

A partir de las micrografías obtenidas por MEB (figura 1), se puede observar que todos los sistemas estudiados AG/Sol-gel2, (c) AG/Sol-gel10 y AG/Sol-gel15, presentaron un recubrimiento uniforme con presencia de algunas discontinuidades en toda la superficie del substrato, además de eso, los sistemas que presentaron mayor regularidad y compatibilidad con el substrato fueron aquellos que se obtuvo con un tiempo mayor de permanencia en el sol, es decir los sistemas AG/Sol-gel10 y AG/Sol-gel15 (figura 1- b y c). La película obtenida con tiempo de permanencia en el sol igual a $2 \mathrm{~min}$ AG/Sol-gel2 (figura 1- a) presentó mayor número de fisuras que comprometió la adherencia de la película al substrato y que puede ser observado por el desplazamiento de la película en ciertas regiones luego del ensayo de polarización (figura 5). Las imágenes obtenidas por MEB, para todos los sistemas estudiados AG/Sol-gel2, AG/Sol-gel10 y AG/Sol-gel15, luego del ensayo de polarización, muestran que el ataque superficial fue más crítico para el sistema AG/Sol-gel2 (figura 4-a), presentando un desplazamiento de la película respecto al substrato. 
a)
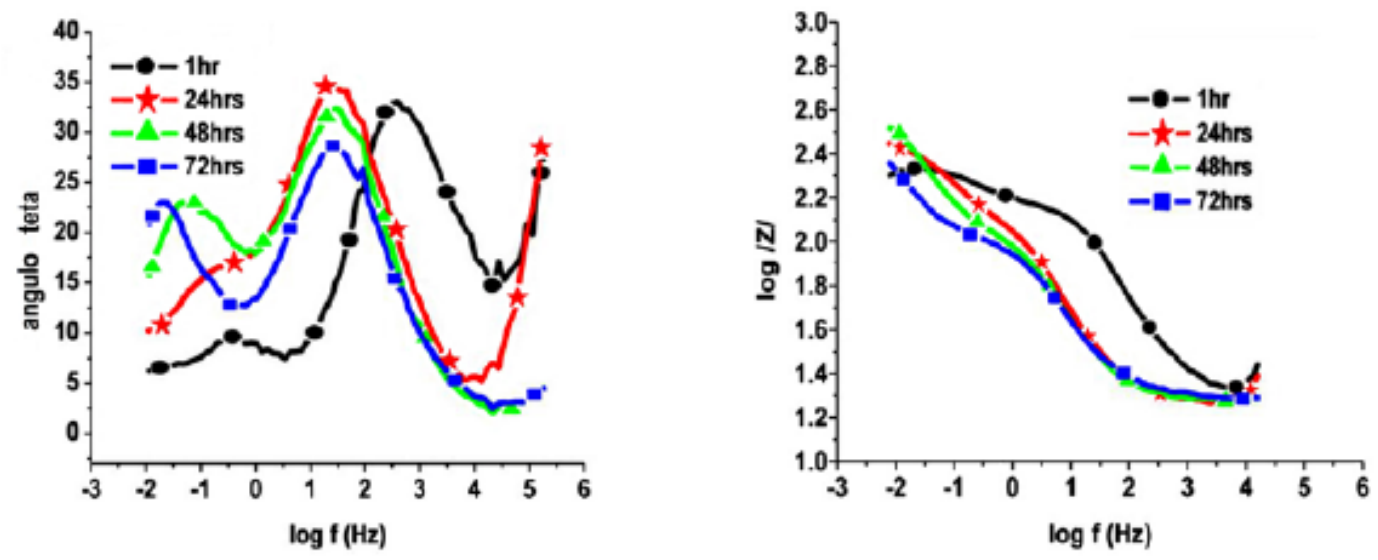

b)
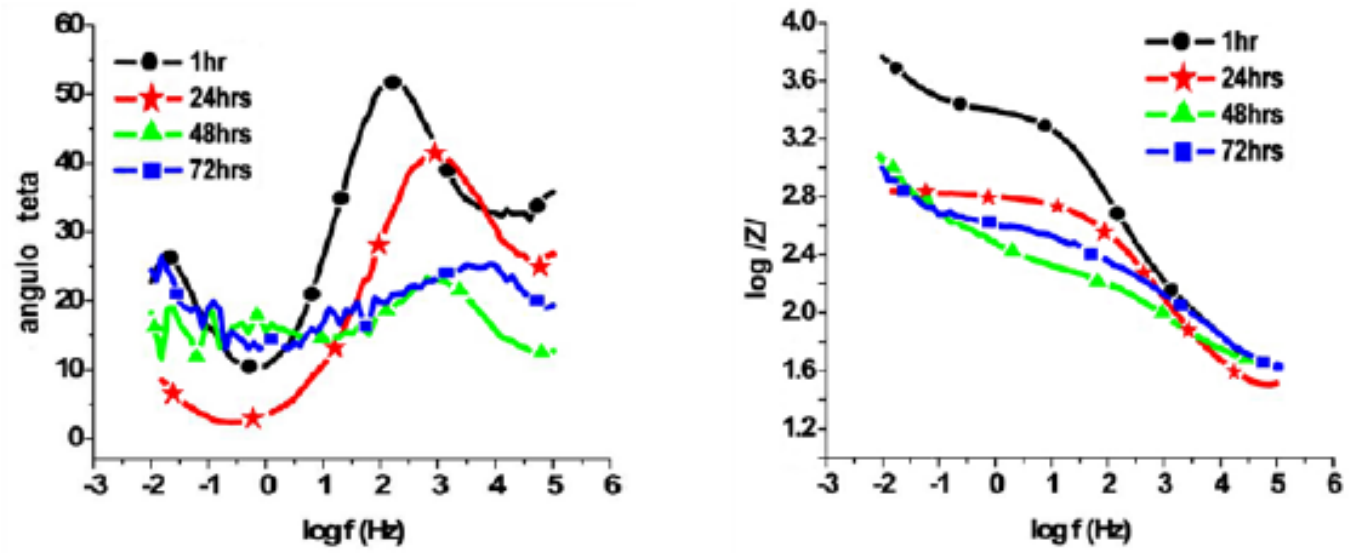

c)
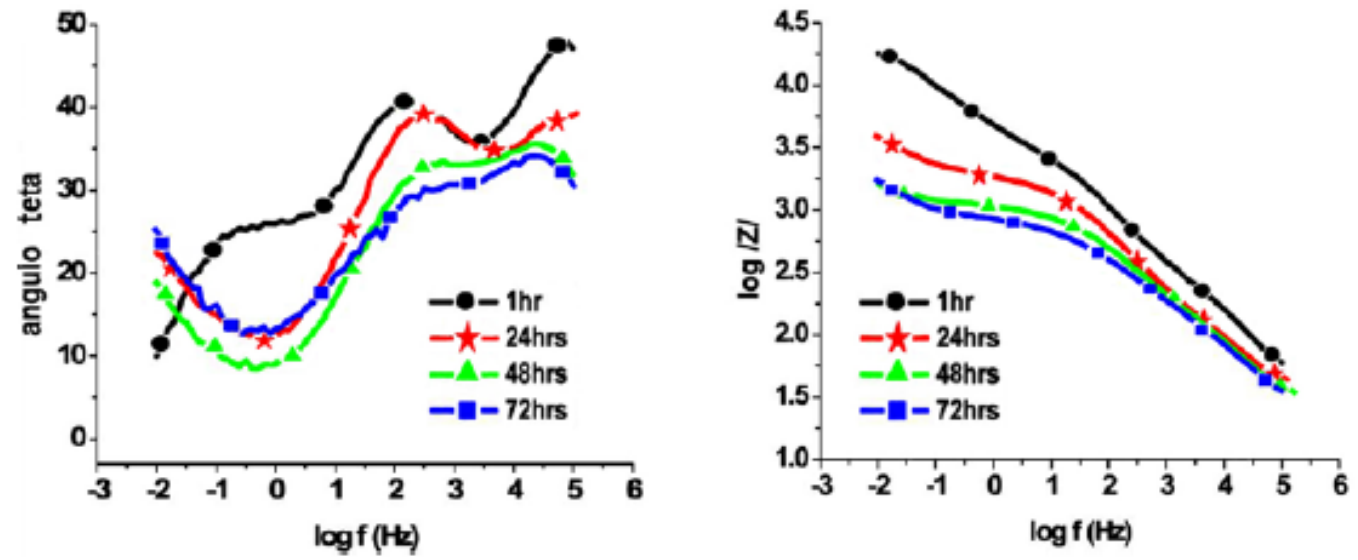

d)
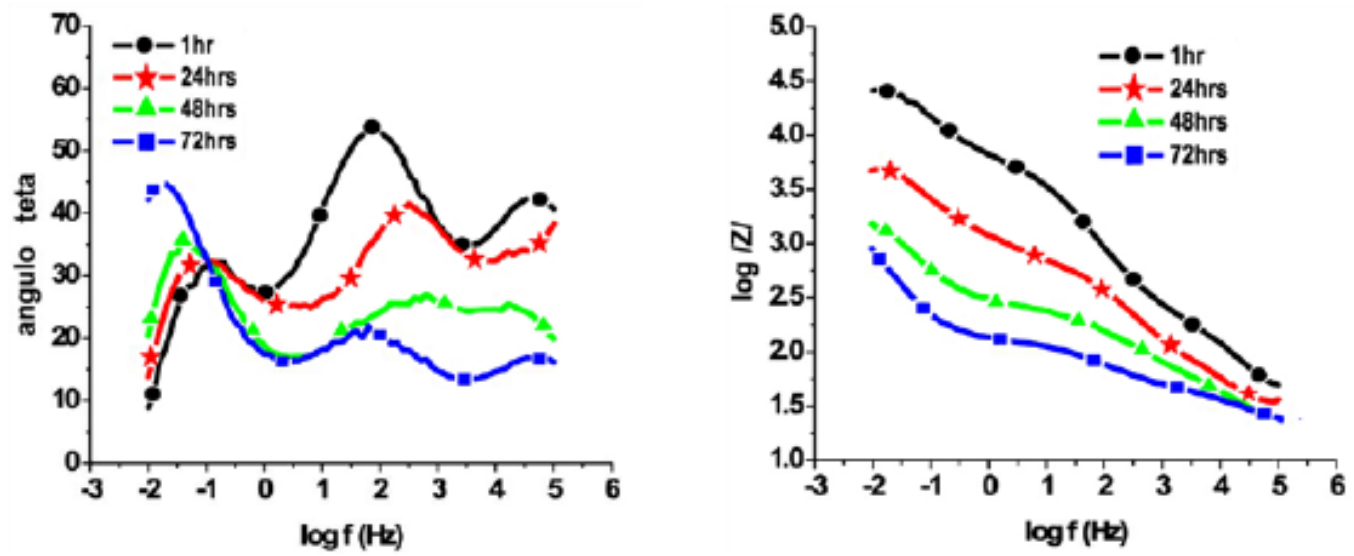

Fig. 6: Diagrama de impedancia versus el tiempo de inmersión en $\mathrm{NaCl} 5 \%$ para los sistemas (a) AG; (b) AG/Sol-gel2; (c) AG/Sol-gel10; (d) AG/Sol-gel15 
Los resultados de los ángulos (figura 2 y tabla 2), para todos los sistemas estudiados AG/Sol-gel2, AG/Sol-gel10 y AG/Sol-gel15, presentaron un ángulo de contacto mayor en relación al acero galvanizado sin revestimiento (AG), lo que indica que la película obtenida redujo la mojabilidad de la superficie, pero también se puede observar que dentro de estos sistemas estudiados, el sistema AG/Sol-gel10 (figura 2- b), obtuvo el mayor ángulo de contacto, indicando una mayor hidrofobicidad con respecto a los demás sistemas. Esto puede estar asociado al hecho de que, ese revestimiento presenta una mayor regularidad y compatibilidad con el substrato, conforme muestran las imágenes obtenidas por MEB (figura 1). Otro detalle importante se observa en el sistema AG/Sol-gel2 que presentó un valor más bajo del ángulo de contacto y por lo tanto una mayor mojabilidad, que esta posiblemente asociado a las fisuras que pueden observarse en las imágenes realizadas por MEB (figura 1) y evaluadas en los valores bajos de resistencia a la polarización que se obtuvo (figura 4). Los valores de los potenciales de circuito abierto (figura 3), para todos los sistemas estudiados quedaron muy próximos de los valores del potencial de circuito abierto del acero galvanizado. Eso se evidencia por que todas las películas obtenidas presentan discontinuidades en la superficie.

Los resultados obtenidos a partir del ensayo de polarización (figura 4 y tabla 3 ) muestran que el pos-tratamiento con la película híbrida promovió el aumento de la resistencia de polarización (Rp) y la disminución de la icorr para todos los sistemas estudiados (tabla 3), evidenciando la acción protectora de esos revestimientos. A partir de los resultados obtenidos por las curvas de polarización no fue posible observar una diferencia significativa entre los revestimientos estudiados, frente al desempeño contra la corrosión, lo que se observó fue que los revestimientos AG/Sol-gel10 y AG/Sol-gel15, fueron los sistemas que obtuvieron el mejor desempeño en relación al otro sistema estudiado (AG/Sol-gel2). Eso evidencia la influencia del tiempo de permanencia en la solución y el efecto positivo de los pos-tratamientos estudiados en la protección contra la corrosión del acero galvanizado, cuando ellos quedan más tiempo inmersos en la solución conteniendo los precursores de silanos (10 y 15 minutos). A partir de los gráficos de impedancia electroquímica obtenidos (figura 6), fue posible observar que el módulo de impedancia es mayor para los sistemas pre-tratados estudiados (figura 6-b,c,d) en comparación al substrato de acero galvanizado sin revestimiento (figura 6-a), demostrando así, la influencia de los pre-tratamientos empleados.

Fue posible verificar de los sistemas estudiados que los revestimientos AG/Sol-gel10 y AG/Solgel15 (figura 6-c, d), fueron los que presentaron los mayores valores de módulo de impedancia con respecto al pre-tratamiento AG/Sol-gel2 (figura 6- a). Eso está asociado al hecho de que los tiempos mayores de permanencia en la solución mejora el desempeño, de la película barrera contra la corrosión, posiblemente, debido a la mejor homogeneidad del revestimiento, conforme se observa en los valores del ángulo de contacto (figura 2 y tabla 2) y en las curvas de polarización obtenidas (figura 4 y tabla 3 ).

Se observa que los sistemas AG/Sol-gel10 y AG/Sol-gel15 (figura 6- c,d), presentaron un alto módulo de impedancia para todos los tiempos de inmersión en $\mathrm{NaCl} 5 \%$ (1, 24, 48 y 72 horas) en relación al acero galvanizado sin revestimiento (figura 6- a), esto muestra el efecto positivo de la película barrera que se forma, conforme se mencionó en los ensayos de polarización y ángulo de contacto, cuando el tiempo de permanencia en la solución es la más adecuado. Mientras que para el sistema AG/Sol-gel2 (figura 6- b), solo en la primera hora de inmersión en el electrolito hubo un mayor valor del módulo de impedancia, que en los otros tiempos de inmersión estudiados, este quedó estabilizado con un valor semejante a aquel que se observó, para el acero galvanizado sin revestimiento (figura 6- a), indicando la baja resistencia de ese revestimiento.

\section{CONCLUSIONES}

Los resultados obtenidos muestran que todos los sistemas estudiados presentan una cobertura regular sobre el acero galvanizado, observándose discontinuidades (fisuras) en la superficie de la película de silano, de acuerdo con la evaluación obtenida por MEB, monitoreando el potencial de circuito abierto y los resultados que se obtuvieron fueron a partir de la técnica de impedancia electroquímica. 
El revestimiento AG/Sol-gel2 presentó una mayor mojabilidad y menor valor del modulo de impedancia con respecto a los demás sistemas, lo que indica que el tiempo de permanencia de 2 minutos en el sol, fue insuficiente para la formación de una capa de barrera protectora, quedando evidenciado el efecto del tiempo de permanencia en el sol, sobre la uniformidad $y$ consecuentemente sobre la resistencia a la corrosión de las películas híbridas que se obtuvieron.

Los resultados indican que a mayores tiempos de permanencia en el sol, permiten la obtención de películas con mayor regularidad de cobertura y consecuentemente, con mejor efecto barrera. Las películas obtenidas con 10 minutos (AG/Sol-gel10) y 15 minutos (AG/Sol-gel15), de permanencia en el sol, presentaron mejor desempeño respecto a la resistencia a la corrosión. De acuerdo con la literatura (Ooij, 2005), fueron realizados análisis hasta un tiempo de inmersión de 30 minutos, del substrato en el sol, siendo el mejor tiempo de permanencia de 15 minutos.

\section{AGRADECIMIENTOS}

El presente trabajo fue realizado con el apoyo de la CAPES, que es la entidad del gobierno Brasilero orientado a la formación de recursos humanos. Los autores agradecen también el apoyo del CNPq, FAPERGS, Centro de Microscopia de la UFRGS, por los análisis de MEB y al Laboratorio de Óptica y Laser del Instituto de Física de la UFRGS, por los análisis del ángulo de contacto.

\section{REFERENCIAS}

Aldykiewicz, A.J. Jr, Davenport A.J. y Isaacs H.S., The Electrochemical Society, p.143-147 (1996)

Aramaki, K., The inhibition effects of cation inhibitors on corrosion of zinc in aerated $0.5 \mathrm{M} \mathrm{NaCl}$ Corrosion Science, v.43, p.1573 (2001)

Arenas, M.A. y Damborenea J.J., Growth mechanisms of cerium layers on galvanized steel Electrochimical Acta v.48, p.3693-3698 (2003).

Bernal, S. y 5 autores más, Lanthanide salts as alternative corrosion inhibitors - The Journal of Alloys and Compounds. p.225 (1995)

Cabral, E.R y Mannheimen E.A. Galvanização, sua aplicação em equipamentos elétricos, Libro técnico s/a, industria y comercio, Rio de Janeiro (1979)

EPA (Environmental Protection Agency), "Toxicological review of hexavalent chromiumre", Disponible en: www.epa.gov Accesado el 15 de diciembre del 2010 (1998)

Ferreira, M.G, Duarte R.G, Montemor, M.F y Simoes A.M.P, Silanes and rare earth salts as chromate replacers forpre-treatments on galvanised steel, Electrochimical Acta, v.49 p.2927-2935, (2004)

Geary, M. y Breslin C. B., The influence of dichromate and cerium passivation treatments on the dissolution of Sn/Zn coatings, Corrosion Science, v.39, p.1341-1350 (1997)

Jones, D.A., Principles and prevention of corrosion, 2nd ed., prentice-hall inc, USA, p. 477 (1996)

Kunst, S.R, Obtenção e caraterização de revestimentos híbridos a base de precursores alcoóxidos sobre o aço galvanizado, Tesis de maestria, Escuela de Ingeniería, Universidade Federal do Rio Grande do Sul, Porto Alegre (2011)

Montemor, M.F, Simoes A.M. y Ferreira M.G.S., Composition and corrosion behaviour of galvanised steel treated with rare-earth salts: the effect of the cation, Progress in Organic Coatings, v.44, p.111-120 (2002)

Montemor, M. F., Trabelsi W., Zheludevich M.L. y Ferreira M. G. S., Progress in organic coatings, v.57 (2006) 
Ooij, V.W.J. y otros 5 autores, Corrosion protection properties of organofunctional silanes - An overview, Science and technology, v.10, p.639-664 (2005)

Trabelsi, W. y otros 4 autores, "An electrochemical and analytical assessment on the early corrosion behavior of galvanized steel pretreated with aminosilanes", Surface \& Coantings Techonology (2004)

Yasakau, K.A, Zheludkevich M.L, Karavai O.V y Ferreira M.G.S; Progress in organic coatings, v.63, p.352-361 (2007) 